Corrigendum

\title{
Corrigendum to "Continuous theta-burst stimulation (cTBS) over the lateral prefrontal cortex alters reinforcement learning bias" [Neuroimage 57 (2) (2011) 617-623]
}

\author{
Derek V.M. Ott ${ }^{\mathrm{a}, \mathrm{e}, *}$, Markus Ullsperger ${ }^{\mathrm{b}, \mathrm{c}}$, Gerhard Jocham ${ }^{\mathrm{b}, \mathrm{d}}$, Jane Neumann ${ }^{\mathrm{e}, \mathrm{f}}$, Tilmann A. Klein ${ }^{\mathrm{e}, *}$ \\ a Dept. of Neurology, University Clinic Leipzig, Germany \\ b Max Planck Institute for Neurological Research, Cologne, Germany \\ c Radboud University Nijmegen, The Netherlands \\ d FMRIB Centre, University of Oxford, UK \\ e Max Planck Institute for Human Cognitive and Brain Sciences, Leipzig, Germany \\ ${ }^{\mathrm{f}}$ Leipzig University Medical Center, IFB Adiposity Diseases, Germany
}

The authors regret having misquoted the article from Ko JH et al. (2008). ("Theta burst stimulation-induced inhibition of dorsolateral prefrontal cortex reveals hemispheric asymmetry in striatal dopamine release during a set-shifting task: a TMS-[(11)C]raclopride PET study") in the present publication. This happened due to a misreading of the paper and led to a partial reversion of the findings reported by Ko et al. Even though this does not affect the overall conclusions derived from the data in Ott et al. (2011), the authors do believe that it is appropriate to re-appraise Ko et al. (2008) in the context of their own manuscript.

Correction 1: Recently, several studies have shown that repetitive transcranial magnetic stimulation (rTMS) applied to the prefrontal cortex can trigger the release of dopamine in both the striatum (Ko et al., 2008; Pogarell et al., 2007, 2006; Strafella et al., 2001) and in distant prefrontal areas (Cho and Strafella, 2009).

Ko et al. (2008) should be removed as a quotation at this point. After this paragraph, the following should be added: Another study, however, has reported a decrease of free dopamine in the striatum after left-hemispherical cTBS (Ko et al., 2008).

Correction 2: A surprising finding has been that stimulation of the left but not the right dorsolateral prefrontal cortex (DLPFC) leads to increased striatal DA (Strafella et al., 2001; Pogarell et al., 2007, 2006) in studies using both positron emission tomography (PET) and single photon emission computed tomography (SPECT). The magnitude of DA release was comparable to chemical challenge with amphetamine (Pogarell et al., 2007) and paralleled by impaired performance in the Montréal Card Sorting Test (MCST), a task requiring cognitive set-shifting (Ko et al., 2008).

This should be rephrased as the following: A surprising finding has been that stimulation of the left but not the right dorsolateral

DOI of original article: http://dx.doi.org/10.1016/j.neuroimage.2011.04.038.

* Corresponding authors at: Max Planck Institute for Human Cognitive and Brain Sciences, Leipzig, Germany.

E-mail addresses: ottd@cbs.mpg.de (D.V.M. Ott), tklein@cbs.mpg.de (T.A. Klein). prefrontal cortex (DLPFC) modulates striatal DA (Strafella et al., 2001; Pogarell et al., 2007, 2006) in studies using both positron emission tomography (PET) and single photon emission computed tomography (SPECT). When dopamine release was increased, the magnitude was comparable to chemical challenge with amphetamine (Pogarell et al., 2007); in the case of decrease, the DA-effect was paralleled by impaired performance in the Montréal Card Sorting Test (MCST), a task requiring cognitive set-shifting (Ko et al., 2008).

Correction 3: In the present study, we applied cTBS to the prefrontal cortex, which had been shown to increase dopamine release in the ventral striatum (Ko et al., 2008; Strafella et al., 2001).

This sentence is not correct in its present form. rTMS (as in Strafella et al., 2001) has been shown to increase dopamine release, whereas cTBS (as in Ko et al., 2008), thus far, hasn't. Therefore, the sentence should be changed accordingly: In the present study, we applied cTBS to the prefrontal cortex, which had been shown to modulate dopamine release in the ventral striatum (Ko et al., 2008; Strafella et al., 2001).

Correction 4: Thus, in our experiment, cTBS to the left DLPFC lead to an enhancement of both reward sensitivity and striatal prediction error coding, which is in agreement with previous findings on [11C]raclopride binding, suggesting an increase in ventro-striatal dopamine release after similar left-prefrontal stimulation (Ko et al., 2008), which is known to enhance approach learning (Frank et al., 2004).

This needs to be rephrased and discussed: Thus, in our experiment, cTBS to the left DLPFC led to an enhancement of both reward sensitivity and striatal prediction error coding, most likely reflecting an increased dopamine effect and enhancing approach learning (Frank et al., 2004). However, the only study thus far measuring dopamine release by [11C]raclopride binding (Ko et al., 2008) found increased binding capacity, reflecting a decrease in synaptic dopamine. The origin of this discrepancy is unknown and might be based on methodological grounds or functional anatomy: Ko et al. used three trains of pulses lasting $20 \mathrm{~s}$ each and separated by 1-minute intervals, adding up to 600 pulses; we applied a single continuous train of $40 \mathrm{~s}$ (400 pulses). Anatomically, Ko et al. stimulated $\mathrm{x}=+/-30$, $\mathrm{y}=40, \mathrm{z}=26$; we stimulated $\mathrm{x}=+/-40, \mathrm{y}=21, \mathrm{z}=27$; these 
locations are likely to be integrated in functionally distinct networks (Petrides, 2005). Finally, the task itself (card-sorting-task vs. probabilistic learning task) may be of relevance.

Correction 5: In some studies, the resulting striatal dopamine release was observed only ipsilaterally (Strafella et al., 2001), in other studies bilaterally (Pogarell et al. 2006; Ko et al., 2008).

Ko et al. (2008) should be removed from the citations and the following sentence should be added: However, one study using cTBS observed an ipsilateral decrease in dopamine release (Ko et al., 2008).

Correction 6: The regions in which activity was modulated by cTBS match nicely with findings from Strafella and colleagues. They described reduced [11C]FLB 457-binding in MOFC and RCZ (Cho and
Strafella, 2009) and reduced [11C]raclopride-binding in the NAcc (Ko et al., 2008), indicative of increased DA release.

This must be rephrased: The regions in which activity was modulated by cTBS match nicely with findings from Strafella and colleagues. They described reduced [11C]FLB 457-binding in mOFC and RCZ (Cho and Strafella, 2009) and increased [11C]raclopride-binding in the NAcc (Ko et al., 2008), indicative of an increase and decrease in DA release, respectively.

Additional reference:

M. Petrides: Lateral prefrontal cortex: architectonic and functional organization. Philos Trans R Soc Lond B Biol Sci, 360 (2005), pp. 781-795.

The authors would like to apologise for any inconvenience caused. 